\title{
Metabolism in pregnancy: a field for novel therapies
}

Nikolaos Vrachnis, Dimitrios Zygouris, Sophia Kalantaridou and Zoe Iliodromiti

In response to the recent Review by Sheila Collins (A heart-adipose tissue connection in the regulation of energy metabolism. Nat. Rev. Endocrinol. 10, 157-163; 2014), ${ }^{1}$ we would like to mention important data pertaining to pregnant women, a special population in whom natriuretic peptides are widely studied. During pregnancy, maternal lipid metabolism changes to a catabolic state characterized by increased adipose tissue lipolysis. ${ }^{2}$ This catabolic state is the target of many pharmaceutical and behavioural treatment interventions.

In the past couple of years, baseline natriuretic peptide B (BNP) levels during pregnancy have been found not to be correlated with maternal pregnant weight or BMI. ${ }^{3}$ This observation demonstrates that, in pregnancy, BNP is produced at elevated levels, regardless of adipose tissue deposits, thus indicating that BNP plays a vital part in maternal metabolism. In women with preeclampsia, BNP levels are correlated with leptin levels, implying a potential role for BNP in lipid metabolism. ${ }^{4}$ The increased lipolysis observed during pregnancy could, therefore, be partly attributed to elevated levels of natriuretic peptides as compared with those in adults who are not pregnant. Equally noteworthy is the fact that natriuretic peptide $A(A N P)$ levels are high in the foetus, indicating the involvement of ANP in increased neonatal thermogenesis. ${ }^{5}$

We thus propose that metabolism during pregnancy could be a model for future therapeutic interventions in other adult groups, pregnant women forming an ideal study group on account of their increased metabolism and BNP levels. Identifying targeted therapies that either augment natriuretic peptide production or sensitize the respective receptors may lead to the alleviation of obesity and cardiometabolic disease.

Finally, further research is likely to elucidate whether pregnancy might have a protective role against obesity and cardiometabolic disease, resulting from increased lipolysis in multiparous compared with nulliparous women.

$2^{\text {nd }}$ Department of Obstetrics and Gynecology, University of Athens Medical School, 124B Vasilissis Sophias Avenue, Athens 11526,
Greece (N.V.). Hellenic Society of Obstetrics and Gynecology, 10 Alkaiou Street, Athens 11528, Greece (D.Z.). Department of Obstetrics and Gynecology, Room 220C, University of loannina Medical School, Panepistimiou Avenue, 45100 Ioannina, Greece (S.K.). Department of Neonatology, University of Athens Medical School, Aretaieio Hospital, 2 Anninou Street, Zografou 15771, Athens, Greece (Z.I.).

Correspondence to: N.V.

nvrachnis@hotmail.com

Competing interests

The authors declare no competing interests.

1. Collins, S. A heart-adipose tissue connection in the regulation of energy metabolism. Nat. Rev. Endocrinol. 10, 157-163 (2014).

2. Martin-Hidalgo, A. et al. Lipoprotein lipase and hormone-sensitive lipase activity and mRNA in rat adipose tissue during pregnancy. Am. J. Physiol. 266, E930-E935 (1994).

3. Yurteri-Kaplan, L. et al. Brain natriuretic peptide in term pregnancy. Reprod. Sci. 19, 520-525 (2012).

4. Masuyama, H., Nobumoto, E., Inoue, S. \& Hiramatsu, Y. Potential interaction of brain natriuretic peptide with hyperadiponectinemia in preeclampsia. Am. J. Physiol. Endocrinol. Metab. 302, E687-E693 (2012).

5. Chevalier, R. L. The moth and the aspen tree: sodium in early postnatal development. Kidney Int. 59, 1617-1625 (2001). 